\title{
Diffuse large B-cell lymphoma presenting with cholecystitis-like symptoms
}

\author{
Sung Pil Yun, Hyung II Seo \\ Department of Surgery, Biomedical Research Institute, Pusan National University Hospital, Busan, Korea
}

Diffuse large B-cell lymphoma that arises from the gallbladder is extremely rare, and the associated studies are not well described in the literature. We report our experience that diffuse large B-cell lymphoma of the gallbladder was diagnosed by histological findings after laparoscopic cholecystectomy in a 75-year-old man. The patient was diagnosed with stage IV lymphoma, and chemotherapy was performed following surgery. The abdominal, chest, neck computed tomography (CT) and positron emission tomography (PET)-CT were performed after chemotherapy, and the results showed that there were no multiple lymphadenopathies. The patient was considered to have achieved complete remission. Diffuse large B-cell lymphoma of the gallbladder is extremely rare and never been diagnosis preoperatively. Pathological examination of the cholecystectomy specimen is important. This will be very helpful for identifying patients who need additional treatment.

Keywords: Diffuse large B-cell lymphoma, Gallbladder, Cholecystitis

\section{INTRODUCTION}

Laparoscopic cholecystectomy has become the standard procedure for the treatment of acute or chronic cholecystitis and it is performed easily. After surgery, according to the histopathological analysis of the biopsy, the postoperative diagnosis can change unlike the preoperative diagnosis. For example, after laparoscopic cholecystectomy, which is performed as a treatment for cholecystitis, gallbladder cancer is confirmed by histopathological findings and then radical cholecystectomy may be needed. In extremely rare cases, evaluation of the resected gallbladder after cholecystectomy for cholecystitis confirms the diagnosis of primary or systemic lymphoma [1-6]. In such cases, additional chemotherapy may be required. We report our experience that diffuse large B-cell lymphoma of the gallbladder was diagnosed by histological findings after laparoscopic cholecystectomy and additional chemotherapy was performed following surgery. The patient had fever

Received: Mar 22, 2018 Accepted: Jun 6, 2018

Correspondence to: Hyung II Seo

Department of Surgery, Biomedical Research Institute, Pusan National University Hospital, 179 Gudeok-ro, Seo-gu, Busan 49241, Korea

Tel: +82-51-240-7238, Fax: +82-51-247-1365

E-mail: seohi71@hanmail.net

Copyright $@$ Korean Society of Surgical Oncology

This is an Open Access article distributed under the terms of the Creative Commons Attribution Non-Commercial License (http://creativecommons.org/licenses/by-nc/4.0) which permits unrestricted non-commercial use, distribution, and reproduction in any medium, provided the original work is properly cited. and right upper quadrant (RUQ) pain. The preoperative diagnosis was gallstones and chronic cholecystitis; however, the final diagnosis was diffuse large B-cell lymphoma of the gallbladder.

\section{CASE REPORT}

A 75-year-old man with fever, RUQ pain and weight loss presented to the local clinic. Abdominal ultrasound showed multiple gallstones and chronic cholecystitis. The patient was transferred to $\mathrm{Pu}-$ san National University Hospital for surgical treatment. His past medical history was type 2 diabetes mellitus and coronary artery disease. Laboratory studies showed elevation of alkaline phosphatase (418 IU/L; normal range, 95-280 IU/L) and lactate dehydrogenase (1,579 IU/L; normal range, 218-472 IU/L), but other data were within normal limits. The abdominal computed tomography (CT) images revealed stones in the gallbladder neck portion (Fig. 1A) and edema between the liver bed and the gallbladder wall (Fig. 1B). On the basis of these radiological findings, the patient was diagnosed with gallstones and chronic cholecystitis, and then we performed elective laparoscopic cholecystectomy. Conventional 3-port laparoscopic cholecystectomy was performed. We found stones in the resected gallbladder, but there was no mass lesion and mucosal lesion in the gallbladder. The postoperative course was uneventful and the patient was discharged on postoperative day 4 . Final pathology of the resected gallbladder was diffuse large B-cell lymphoma. Highly pleomorphic cells were present in the vascular 

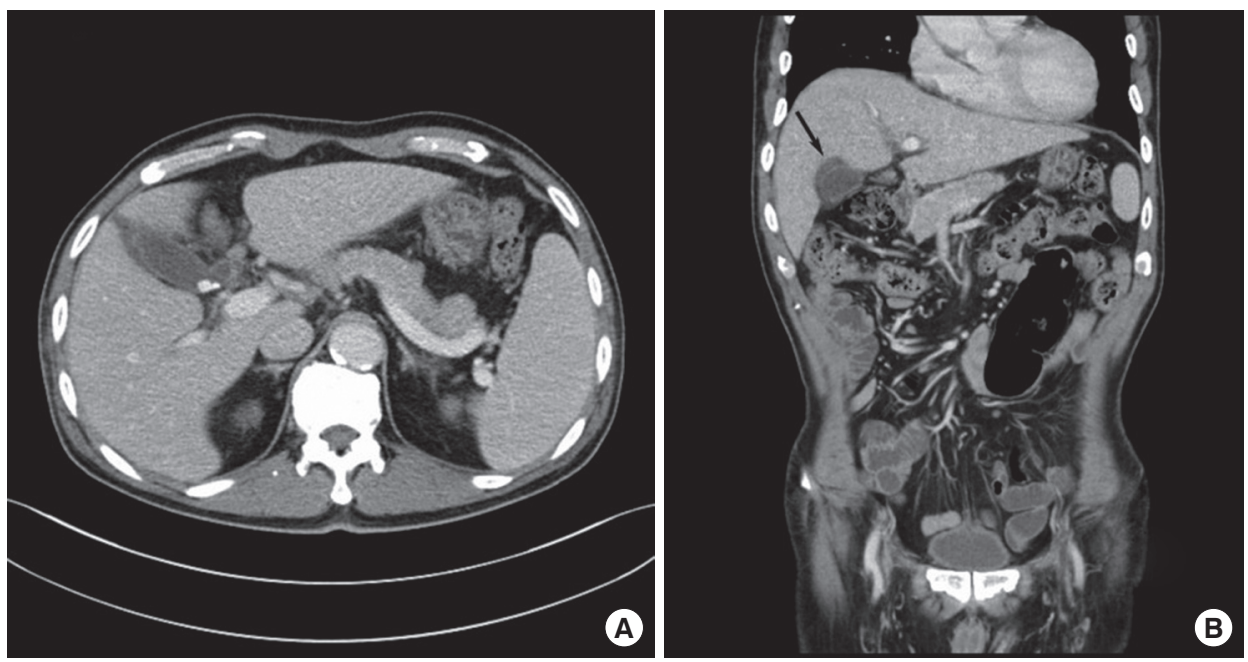

Fig. 1. Preoperative computed tomography (CT) findings. (A) Contrast enhanced abdominal CT demonstrates calcified stones within the gallbladder neck portion. (B) Coronal view shows gallbladder wall edema (arrow).
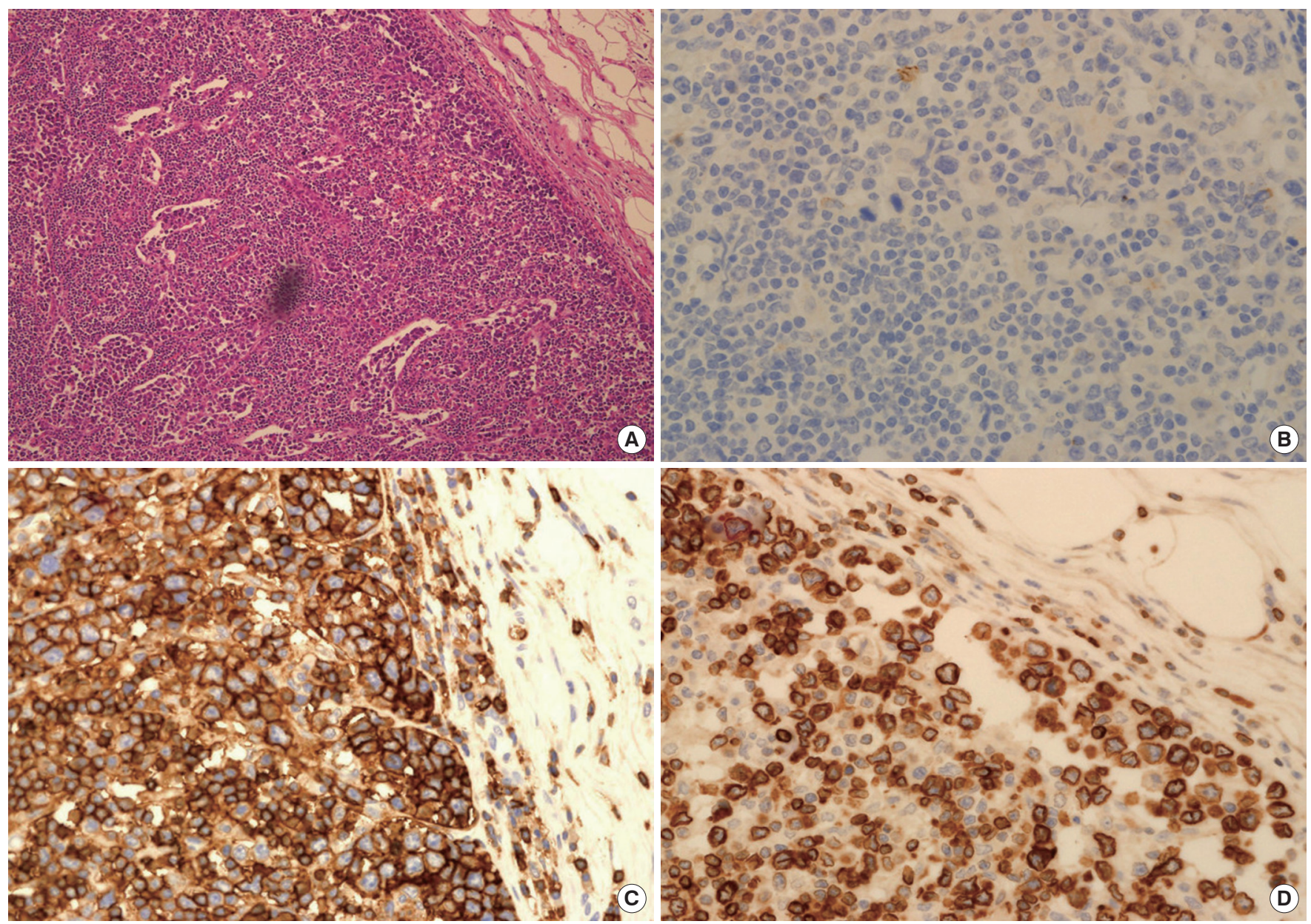

Fig. 2. Histopathological findings. (A) Highly pleomorphic cells are present in the vascular space in the subserosa of the gallbladder and in the sinus of adjacent lymph node. The cells are large, pleomorphic, and sometimes anaplastic ( $\times 200, H \& E$ stain). (B) On immunohistochemical staining, tumor cells are negative for epithelial membrane antigen $(\times 400)$. (C, D) Tumor cells are positive for leukocyte common antigen $(C, \times 400)$ and $B c l-2(D, \times 400)$. Therefore, final pathology was diffuse large B-cell lymphoma. 

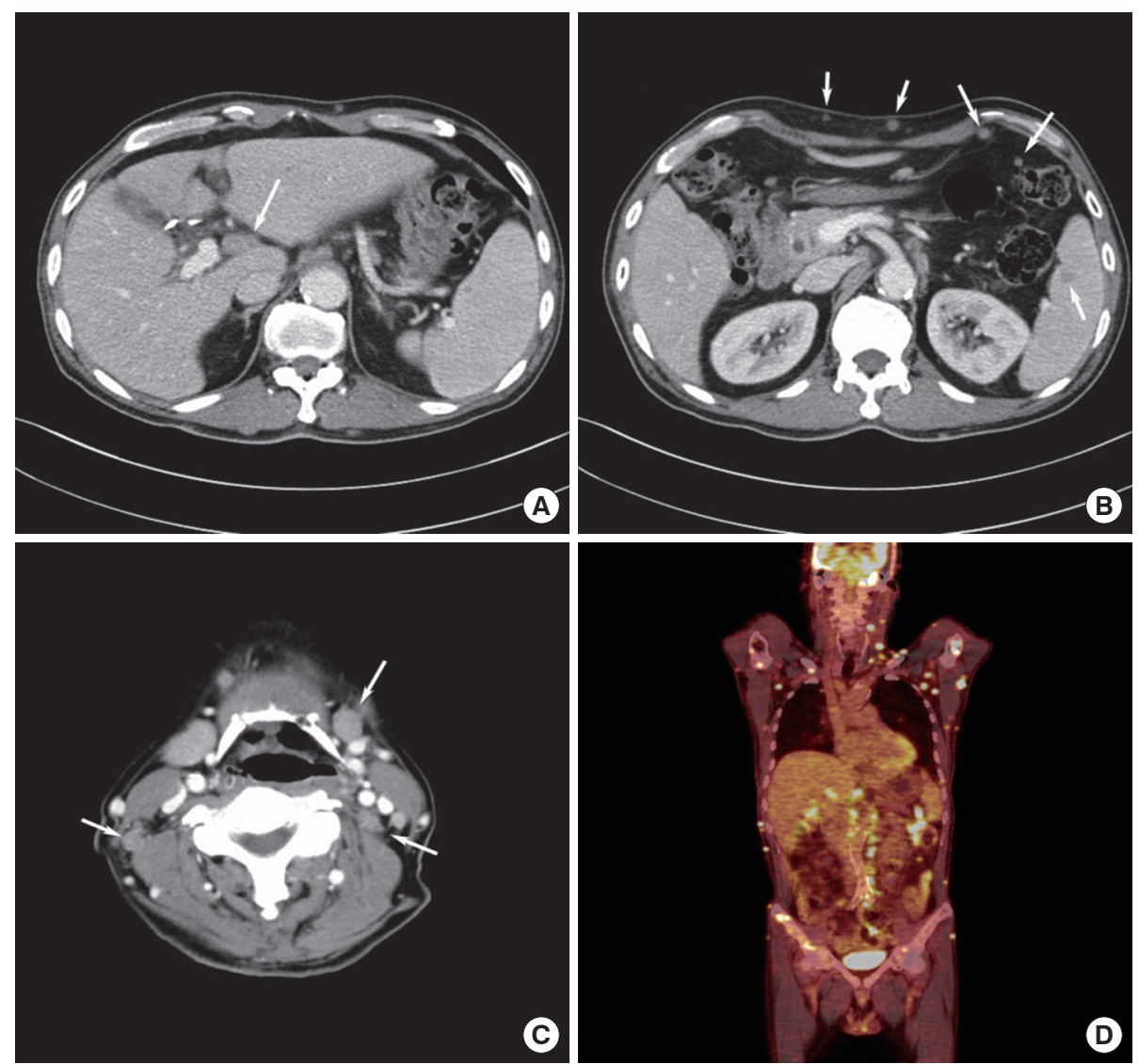

Fig. 3. After 2 months, multiple lymphadenopathies were observed on contrasted-enhanced abdominal, neck computed tomography (CT) and positron emission tomography (PET)-CT. (A) Abdominal CT demonstrates lymph node enlargement in the hepatoduodenal ligament (arrow). (B) Multiple lymph node enlargement in skin, spleen, peritoneum (arrows). (C) Neck CT demonstrates multiple lymph node enlargement in bilateral neck level IA, IB, left II and IV, right VA (arrows). (D) PET-CT shows lymphoma involvement of bilateral neck, SCN, bilateral axillary, mediastinal, diaphragmatic, hepatoduodenal, celiac trunk, SMA root, retroperitoneal, retrocrural, bilateral common iliac, splenic, and subcutaneous lymph nodes in the whole body and multiple bones. SCN, supraclavicular lymph node; SMA, superior mesenteric artery.

space in the subserosa of the gallbladder and in sinus of the adjacent lymph node. The cells were large, pleomorphic, and sometimes anaplastic, being positive for leukocyte common antigen, CD20, MUM1, and Bcl-2 and negative for CD3, CD45RO, CD5, CD30, epithelial membrane antigen, and anaplastic lymphoma kinase (ALK). Ki-67 index was high up to $70 \%$ to $80 \%$ (Fig. 2). The patient was referred to the hematology-oncology department for evaluation and staging of lymphoma. The tests were performed 2 weeks after the operation with abdominal, chest, neck CT, and positron emission tomography (PET)-CT. The test demonstrated lymph node enlargement in the hepatoduodenal ligament, skin, spleen, and peritoneum. Also, the lymph nodes were founded at bilateral neck levels IA, IB, left II and IV, right VA. PET-CT showed lymphoma involvement of bilateral neck, supraclavicular lymph node, bilateral axillary, mediastinal, diaphragmatic, hepatoduodenal, celiac trunk, superior mesenteric artery root, retroperitoneal, retrocrural, bilateral common iliac, splenic, and subcutaneous lymph nodes in the whole body and multiple bones. The patient was diagnosed with stage IV lymphoma, and chemotherapy was performed. Six cycles of R-CHOP (rituximab, cyclophosphamide, doxorubicin, vincristine, prednisolone) chemotherapy were performed. The abdominal, chest, neck CT and PET-CT were performed after chemotherapy, and the results showed that there were no multiple lymphadenopathies (Fig. 3). Therefore, the patient was considered to have achieved complete remission.

\section{DISCUSSION}

The patient with gallstones and cholecystitis usually presents with RUQ pain, tenderness, fever, and nonspecific symptoms. This disease is confirmed by abdominal ultrasonography and CT, and laparoscopic cholecystectomy is the treatment of choice [7]. Biopsy of 
the resected gallbladder is performed, and finally a histopathological diagnosis is made. Rarely, according to the histopathological analysis of the biopsy, the postoperative diagnosis can change unlike the preoperative diagnosis, and additional treatment may be needed. Generally, a malignant tumor that arises from the gallbladder requires additional treatment, and according to the stage of cancer, re-operation is necessary. Some patients with systemic disease undergo laparoscopic cholecystectomy as the condition is misdiagnosed as benign gallbladder disease with RUQ discomfort.

Primary extranodal presentation of non-Hodgkin lymphoma (NHL) occurs in up to $50 \%$ of cases with gastrointestinal tract being most commonly involved. Gallbladder involvement of lymphoma has been rarely reported, and the pathogenesis is unknown. In the previous report, $42 \%$ of GB-NHL (NHL of the gallbladder) were known to have gallstones with the histology type spanning from mucosa-associated lymphoid tissue (MALT) lymphoma to diffuse large B-cell lymphoma, also, the symptoms of MALT lymphoma and large B-cell lymphoma are similar to those of cholecystitis [1]. Pathological examination of the cholecystectomy specimen is important, especially for those with gallstones, as there is a strong association between gallstones and lymphoma of the gallbladder. Most GB-NHLs are non-MALT lymphomas with aggressive behavior and adjuvant chemotherapy is highly recommended.

In this case, the patient was initially diagnosed with gallstones and cholecystitis. After laparoscopic cholecystectomy, diffuse large B-cell lymphoma of the gallbladder was diagnosed by histological findings. Malignant lymphoma that arises from the gallbladder is extremely rare, and the reported incidence is $0.1 \%$ to $0.2 \%$. According to the report by Ono et al. [4], diffuse large B cell lymphoma of the gallbladder, which is a high-grade lymphoma, mostly presents as a solid and bulky mass in the gallbladder [4]. Mani et al. [3] reported 19 cases of lymphoma of the gallbladder and extrahepatic bile ducts, and 13 patients were diagnosed with primary lymphoma of the gallbladder and five patients were diagnosed with systemic lymphoma on further evaluation. In our case, there was no mass-like lesion in the gallbladder, and lymphoma was detected only in the subserosa and lymph nodes adjacent to the gallbladder. Furthermore, the tests that were performed 2 weeks after the operation, with abdominal, chest, neck CT and PET-CT showed multiple lymphadenopathies which may not indicate primary lymphoma of the gallbladder, but gallbladder and regional lymph node involvement of systemic lymphoma. However, Kato et al. [2] reported primary lymphoma of the gallbladder with only gallbladder wall thickening. In this case, since the initial abdominal CT showed only gallbladder wall thickening with no intra-abdominal lymph node enlargement, we could not rule out primary lymphoma of the gallbladder.
Among the patients who were diagnosed with lymphoma of the gallbladder after cholecystectomy, $42 \%$ had cholelithiasis, and epigastric and RUQ pain were the main symptoms. Also, if there is no mass-like lesion, lymphoma of the gallbladder is diagnosed after surgery, and the patient is usually diagnosed with cholecystitis before surgery $[1,4]$. In our case, the patient underwent cholecystectomy because of gallstones, RUQ pain, and fever, which are seen in cholecystitis. But, symptoms such as fever and weight loss that develop for the first time could be considered as typical B symptoms of lymphoma.

With respect to the postoperative treatment, Yokoe et al. [8] reported that only $27.7 \%$ of the patients who were diagnosed with lymphoma of the gallbladder underwent postoperative chemotherapy. Kato et al. [2] reported the 38-month disease-free survival rate after R-CHOP chemotherapy. Considering that the complete remission rate of NHL after chemotherapy is very high, and the 5 -year survival rate of stage IV NHL is approximately 50\%, aggressive chemotherapy is needed after determining the correct stage of lymphoma through further postoperative evaluation. In our study, the patient was diagnosed as having stage IV lymphoma with aggravated findings during postoperative evaluation, but after six cycles of R-CHOP chemotherapy, he achieved complete remission and maintained a disease free state.

Darmas et al. [9] claimed that a routine histological examination after surgery is valuable only if the patient has a macroscopically abnormal gallbladder. But in our case, there is a possibility that the macroscopically normal gallbladder had not only gallbladder cancer but also some other disease. In conclusion, we think it is very important that biopsy of a resected gallbladder should be performed and histopathological diagnosis should be established, and this will be very helpful for identifying patients who need additional treatment.

\section{CONFLICT OF INTEREST}

No potential conflict of interest relevant to this article was reported.

\section{ACKNOWLEDGMENTS}

This work was supported by clinical research grant from Pusan National University Hospital 2018.

\section{REFERENCES}

1. Huang WT, Chuang SS, Huang CC, Lu CL, Eng HL. Primary diffuse large B-cell lymphoma of the gallbladder with cholelithiasis masquerading as acute cholecystitis: case report and literature re- 
view. NZ Med J 2007;120:U2470.

2. Kato H, Naganuma T, Iizawa Y, Kitagawa M, Tanaka M, Isaji S. Primary non-Hodgkin's lymphoma of the gallbladder diagnosed by laparoscopic cholecystectomy. J Hepatobiliary Pancreat Surg 2008; 15:659-63.

3. Mani H, Climent F, Colomo L, Pittaluga S, Raffeld M, Jaffe ES. Gall bladder and extrahepatic bile duct lymphomas: clinicopathological observations and biological implications. Am J Surg Pathol 2010; 34:1277-86.

4. Ono A, Tanoue S, Yamada Y, Takaji Y, Okada F, Matsumoto S, et al. Primary malignant lymphoma of the gallbladder: a case report and literature review. Br J Radiol 2009;82:e15-9.

5. Shah KG, Molmenti EP, Idrovo JP, Nicastro J, Coppa G. Primary gallbladder lymphoma. Am Surg 2011;77:360-1.

6. Viswanathan SR, Khalpey Z, Ashley SW. Gallbladder lymphoma. Med Oncol 2011;28:810-2.

7. Cameron JL, Gadacz TR. Laparoscopic cholecystectomy. Ann Surg 1991;213:1-2.

8. Yokoe M, Mizuno Y, Hattori K, Kanakura M, Nishi Y, Kumada K, et al. A case of malignant lymphoma of the gallbladder. Nihon Shokakibyo Gakkai Zasshi 2003;100:1219-23.

9. Darmas B, Mahmud S, Abbas A, Baker AL. Is there any justification for the routine histological examination of straightforward cholecystectomy specimens? Ann R Coll Surg Engl 2007;89:23841. 